\title{
A Macroscopic Examination of M. Biceps Femoris and M. Gluteus Maximus in the Orangutan
}

\author{
Misato KASEDA ${ }^{1)}$, Michiko NAKAMURA ${ }^{1)}$, Nobutsune ICHIHARA ${ }^{1)}$, Toshiyuki HAYAKAWA ${ }^{2)}$ and Masao ASARI ${ }^{1) *}$ \\ ${ }^{1)}$ Department of Anatomy 1st, Azabu University School of Veterinary Medicine, 1-17-71 Fuchinobe, Sagamihara, Kanagawa $229-8501$ \\ and ${ }^{2)}$ Department of Anatomy 1st, Jikei University School of Medicine, 3-25-8 Nishi-shinbashi, Minato-ku, Tokyo 105-8461, Japan
}

(Received 26 June 2007/Accepted 6 November 2007)

\begin{abstract}
The musculature of the hip and thigh in the orangutan has been described previously. Anatomically, there are various descriptions among primates in those structures, in particular, the relationship between M. biceps femoris and M. gluteus maximus, their derivatives, and the muscle segment. However, a detailed innervation system to this ischiofemoral part has not been described, thus there is still uncertainty as to with which muscle it is associated. In this analysis, we examined the gross anatomy of the hip and thigh muscles of the orangutan and chimpanzee, including their innervation. Also, a comparison was made with documented data of other primates. As a result of these observations, it was found that the ischiofemoral part in the orangutan is innervated by the same sciatic nerve branch (the common peroneal nerve) as the long head of M. biceps femoris, but not by the same nerve as M. gluteus maximus. Therefore, the ischiofemoral part is appropriately considered as a part of the long head of M. biceps femoris. It appears that this morphologic feature is an adjustment to the arboreal life of the orangutan. The development of the flexor complex of the thigh is necessary for this arboreal adaptation, resulting in a unique musculature of $\mathrm{M}$. biceps femoris in the orangutan.

KEY WORDS: gross anatomy, innervation system, M. biceps femoris, M. gluteus maximus, orangutan.
\end{abstract}

J. Vet. Med. Sci. 70(3): 217-222, 2008

The orangutan (Pongo pygmaeus), the only great ape in Asia, is the only arboreal great ape in the world. It rarely descends to the ground, living most of its life in trees 20-60 $\mathrm{m}$ in height. The orangutan moves from branch to branch utilizing all of its limbs in a unique locomotion system called brachiation. It therefore has a distinctive foot structure that adjusts to the type of grip required. It has a plantigrade foot posture, yet only the exterior of the sole touches the ground. When walking on the ground level, it does not stand with its trunk in a vertical position, and moves with its knees flexed and its lower extremities abducted.

The musculature of the hip and thigh in the orangutan has been previously described, in particular, the relationship of M. biceps femoris and M. gluteus maximus (M. gluteus superficialis). The muscle, around which the present discussion is centered, arises from the ischial tuberosity and inserts onto the shaft of the femur. Hereafter, this is referred to as the ischiofemoral part. Sonntag [7] referred to this ischiofemoral part as the inferior part of M. gluteus maximus, and thus considered it part of M. gluteus maximus. In recent years, Hamada [2] reported that it is not clear to which muscle this ischiofemoral part belongs (M. gluteus maximus, Sigmon, [6]; M. biceps femoris, Stern, [8]). Tuttle and Cortright [10] described the hip musculature of the orangutan as representing a unique pattern amongst the Hominoidae; the lower portion of the $\mathrm{M}$. gluteus maximus complex is a discrete muscle, M. ischiofemoralis. They demonstrate that the ischiofemoral part of the M. ischiofemoralis is an extensor of the hip joint by means of electromyo-

\footnotetext{
* Correspondence to: Dr. Asari, M., Professor, Department of Anatomy 1st, Azabu University School of Veterinary Medicine, 1-17-71 Fuchinobe, Sagamihara, Kanagawa 229-8501, Japan. e-mail: asari@azabu-u.ac.jp
}

graphic studies, and assumed it as an independent muscle of the hip. However, in these reports, a detailed innervation system to this ischiofemoral part has not been described, thus there is still uncertainty as to which muscle this part is associated. In old world monkeys, the part running from the ischial tuberosity to the outside of the thigh is considered to be an independent muscle in the long head of M. biceps femoris [9]. Therefore, it is thought that the relationship of this ischiofemoral part in the orangutan may be closer to $\mathrm{M}$. biceps femoris than $\mathrm{M}$. gluteus maximus.

Fick [1] described the sacrotuberous ligament is absent in this species. However, Sonntag [7] described that the ligament is present, and that M. gluteus maximus originated from it. Whereas, there is no strong evidence either way that M. gluteus maximus originated from the sacrotuberous ligament in the orangutan $[2,5,8]$.

We had the opportunity to study an orangutan, and examine the gross anatomy of hip and thigh muscles, including their innervation. So, we attempted to clarify the form of $\mathrm{M}$. biceps femoris and M. gluteus maximus, especially the ischiofemoral part of each muscle, and also examined the innervation of the muscules derived from the composition of the lumbar and sacral plexus. A comparative anatomical study was also carried out between primates, orangutans and chimpanzee. A comparison was also made with documented data of the anatomy of the baboon (an old world monkey), the gibbon (a small ape in Asia), the gorilla (an African great ape) and the human.

\section{MATERIALS AND METHODS}

In this study, we used a male and a female dead body of the orangutans and a male dead body of the chimpanzee. 
The orangutans were donated from the Yagiyama Zoological Garden (Sendai, Japan). The male individual is 18 years old and was dead by interstitial myocarditis. The female individual is 21 years old and was dead by nephric failure. The chimpanzee was immature and donated from the Keihin Birds and Beasts Trade Ltd (Yokohama, Japan). A comparative study of the gross anatomy of the hip and thigh muscles was carried out between the orangutan and chimpanzee (the African half-arboreal ape).

\section{RESULTS}

Musculature of the Hip and Thigh: In the orangutan, the ischiofemoral part under discussion consideration arose from the ischial tuberosity with the long head of M. biceps femoris, parted from the long head distally and inserted on the lateral lip of the linea aspera of the femur (Figs. 1A, B). The long head inserted at the head of the fibula and the lateral patellar retinaculum, and both the ischiofemoral part and the long head were well developed. There was no connection between the long head and short head of $\mathrm{M}$. biceps femoris (Fig. 1C). The short head was flat and arose from the gluteal tuberosity and between the lateral and medial lips of the linea aspera of the femur and inserted at the head of fibula, the origin tendon of the crural lateral flexor and the crural fascia.

M. gluteus maximus arose from the caudal 1/3 lateral margin of the sacrum and the lateral margin of the coccyx and inserted on the iliotibial tract and the dorsal aspect of the femur (gluteal tuberosity). The sacrotuberous ligament was not observed under M. gluteus maximus (Fig. 1G). Instead of it, a strong membrane of connective tissue was observed between M. gluteus maximus and ischial tuberosity.

In the chimpanzee, the long head of M. biceps femoris arose from the anterior border of the ischial tuberosity, and the long head and short head united in the distal part of the thigh, forming the biceps tendon. The short head arose from the lateral lip of the linea aspera of the femur. The tendon inserted at the lateral aspect of the tibial tuberosity and the crural fascia and did not insert on the head of the fibula (Figs. 2A, D).

M. gluteus maximus arose from the dorsal aspect of the sacrum and coccyx, the sacrotuberous ligament and the ischial tuberosity and inserted at the gluteal tuberosity, the lateral lip of linea aspera of the femur and the lateral patellar retinaculum. In the chimpanzee, the posterior part of $\mathrm{M}$. gluteus maximus appeared to correspond to the ischiofemoral part that had been discussed in the orangutan, and it arose from the ischial tuberosity and inserted at the lateral patellar retinaculum of the femur. The anterior border of the posterior part of M. gluteus maximus formed the intermuscular septa of the lateral thigh contacting M. vastus lateralis, with the superior $2 / 3$ of the posterior border touching the long head and the inferior $1 / 3$ touching the short head. Additionally, the sacrotuberous ligament was observed in the chimpanzee.

Innervation of the Hip and Thigh: In the orangutan, a nerve branch of the sciatic nerve (the common peroneal nerve) in the proximal thigh entered the muscle from the medial aspect of the ischiofemoral part. It also entered the long head of M. biceps femoris (Figs. 1B, C, D). In addition, both $\mathrm{M}$. semitendinosus were also innervated by the same sciatic nerve branch. In the distal thigh, a nerve branch of the common peroneal nerve ran medially to the short head and entered the long head near the insertion. In addition, other nerve branches of the common peroneal nerve entered the short head from the medial aspect (Figs. $1 \mathrm{E}, \mathrm{F})$. In short, in the proximal thigh, the same nerve branch of the sciatic nerve innervated two muscle parts; the ischiofemoral part and the long head of M. biceps femoris. This nerve branch of the sciatic nerve (the common peroneal nerve) was composed of the ventral nerve fibers of L4 and $\mathrm{S} 1$ in the lumbar and sacral plexus, mainly L4 (Fig. 3A). M. gluteus maximus was innervated by the inferior gluteal nerve (the posterior gluteal nerve) and partially by the superior gluteal nerve (the anterior gluteal nerve) (Figs. 1G, H).

In the orangutan, the superior gluteal nerve was composed of the dorsal nerve fibers of L4 and S1 (mainly L4) in the lumbar and sacral plexus. The inferior gluteal nerve was composed of the dorsal nerve fibers of L4, S1 and S2 (mainly S1), and the sciatic nerve was composed of the ventral nerve fibers of L3, L4, S1 and S2 (mainly L4) (Fig. 3A).

In the chimpanzee, the long head of $\mathrm{M}$. biceps femoris was innervated by a branch of the sciatic nerve (the common peroneal nerve) and a branch of the inferior gluteal nerve; the short head was innervated by another branch of the sciatic nerve (a branch of the common peroneal nerve) (Figs. 2C, D). M. semitendinosus, M. semimembranosus and the posterior part of $\mathrm{M}$. gluteus maximus were all innervated by the same sciatic nerve branch as the long head as well. This nerve branch of the sciatic nerve (the common peroneal nerve) was composed of the ventral nerve fibers of $\mathrm{L} 3$ in the lumbar and sacral plexus (Fig. 3B). The posterior part of M. gluteus maximus was innervated by the same branch of the sciatic nerve (the common peroneal nerve) and the same branch of the inferior gluteal nerve as the long head (Figs. 2A, B).

The superior gluteal nerve was composed of the dorsal nerve fibers of L3 and L4 (mainly L3) in the lumbar and sacral plexus. The inferior gluteal nerve was composed of the dorsal nerve fibers of L4, S1 and S2 (mainly L4), and the sciatic nerve was composed of the ventral nerve fibers of L3, L4, S1 and S2 (mainly L4) (Fig. 3B).

\section{DISCUSSION}

The aim of the present study is to classify the musculature of the hip and thigh of the some species of primates, based on the aspect of the innervation. Our gross anatomical observation demonstrates that the ischiofemoral part is innervated by the same sciatic nerve branch as the long head of M. biceps femoris, M. semitendinosus and M. semimembranosus, but not by the same nerves as M. gluteus maximus. Therefore, it is appropriately that this ischiofemoral 

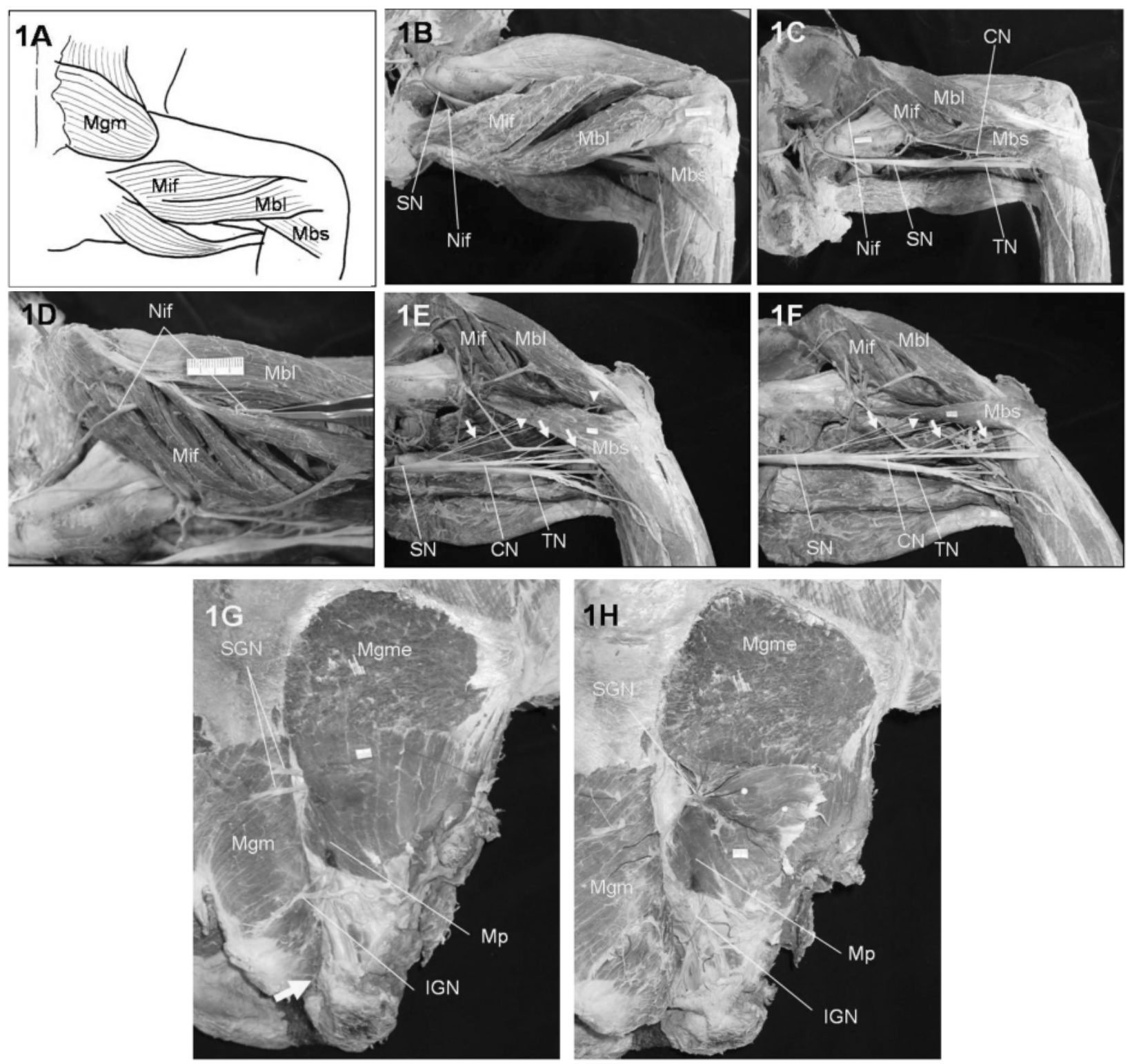

Fig. 1. (A) Schematic drawing of lateral hip and thigh muscles in the orangutan. (B) The lateral aspect of the right thigh in the orangutan. (C) The long head of M. biceps femoris of Fig. 1B was reflected up. (D) Photograph enlarged a part of Fig. 1C. (E) The distal part of the right thigh in the orangutan. (F) The short head of M. biceps femoris of Fig. 1E was reflected up. A nerve branch of the common peroneal nerve (arrowhead) to Mbl. nerve branches of the common peroneal nerve (arrows) to Mbs. (G) The dorsal aspect of the right hip in the orangutan. Instead of the sacrotuberous ligament, a strong membrane of connective tissue was observed between M. gluteus maximus and ischial tuberosity (arrow). (H) M. gluteus medius of Fig. 1G was reflected up. Mif: the ischiofemoral part that arises from the ischial tuberosity and inserts onto the shaft of the femur, Mbl: the long head of M. biceps femoris, Mbs: the short head of M. biceps femoris, Mgm: M. gluteus maximus, Mgme: M. gluteus medius, Mp: M. piriformis, Nif: a nerve branch of the sciatic nerve (the common peroneal nerve) to Mif and Mbl, SN: the sciatic nerve, CN: the common peroneal nerve, TN: the tibial nerve, SGN: the superior gluteal nerve, IGN: the inferior gluteal nerve.

part consisted with a part of the long head of M. biceps femoris, but not be considered a part of $\mathrm{M}$. gluteus maximus.

In old world monkeys, including the baboon, M. biceps femoris has only the long head arising from the ischial tuberosity, but not have a short head. Moreover, the long head of the old world monkeys is separated into two parts distally, and one part, extending from proximal to the ischial tuberosity to the lateral thigh, is considered to be an independent muscle (ischiofemoralis) [9]. On the other hand, there is the short head in M. biceps femoris of the gibbon, the chimpanzee [2], the gorilla [4], and humans [3]. Thus, there is peculiar in dividing pattern of the head of the $M$. 

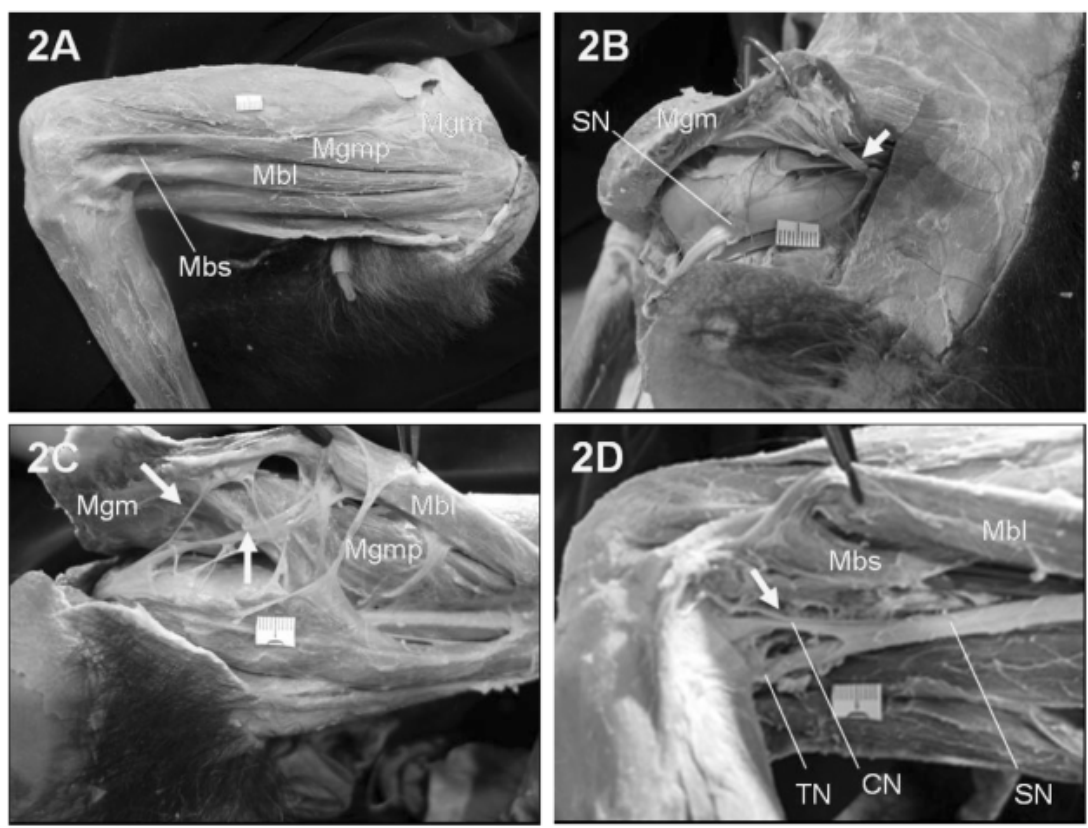

Fig. 2. (A) The lateral aspect of the left thigh in the chimpanzee. (B) The lateral aspect of the left hip in the chimpanzee. The inferior gluteal nerve entered into Mgm (arrow). (C) The lateral aspect of the right thigh in the chimpanzee. The inferior gluteal nerve entered Mgm and Mbl (arrow). (D) The distal part of the left thigh in the chimpanzee. A branch of the sciatic nerve entered Mbs (arrow). Mgm: M. gluteus maximus, Mgmp: the posterior part of M. gluteus maximus, Mbl: the long head of M. biceps femoris, Mbs: the short head of M. biceps femoris, $\mathrm{SN}$ : the sciatic nerve, $\mathrm{CN}$ : the common peroneal nerve, $\mathrm{TN}$ : the tibial nerve.
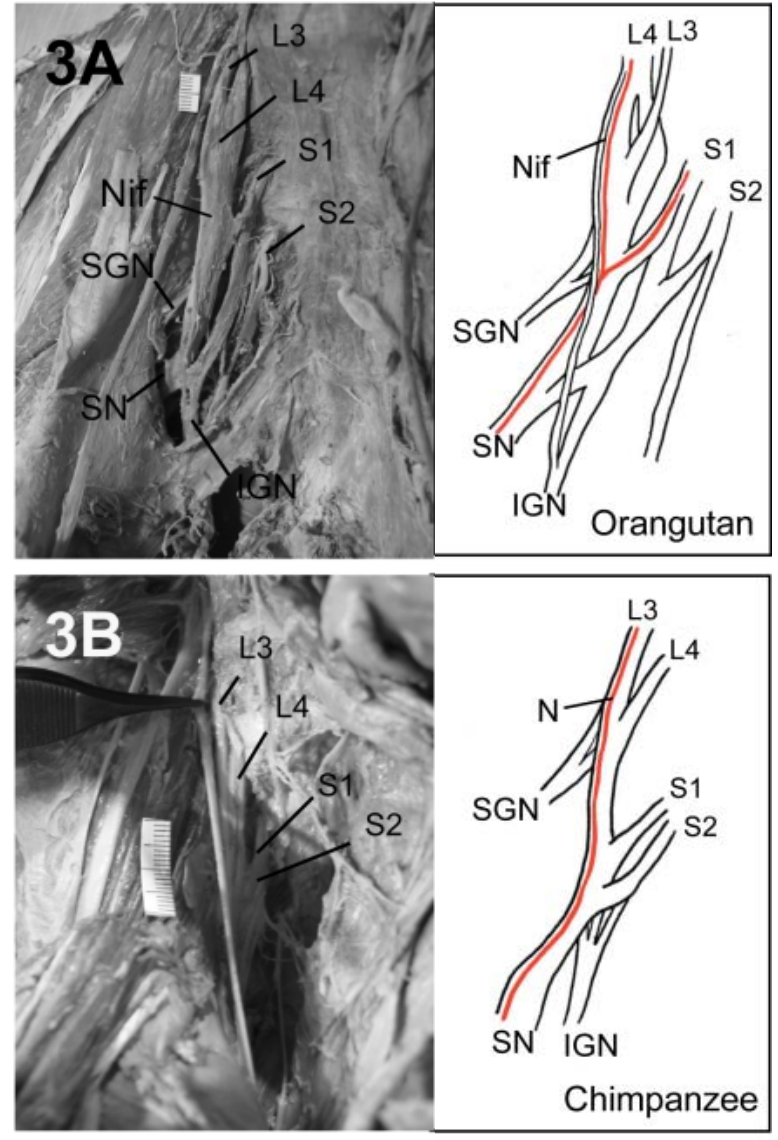

bicepts femorialis in primete.

In the present study, it was found that the long head and short head united in the distal part in the chimpanzee. Due to the previous description that a part of M. gluteus maximus (the posterior part) inserts in the distal thigh in the gibbon (ischiofemoral part [2]), the chimpanzee, and the gorilla [4], it is thought that this posterior part of M. gluteus maximus corresponds to the ischiofemoral part that has been demonstrated in the orangutan.

Hamada [2] also describes that, M. gluteus maximus is separated into anterior and posterior parts, and M. biceps femoris (M. ischiocruralis lateralis) is separated into femoral and crural parts in the old world monkeys. In apes other than the orangutan, M. gluteus maximus is separated into anterior, middle and posterior (ischiofemoral) parts, and $\mathrm{M}$. biceps femoris is separated into long and short heads. While in humans, there is no part that corresponds to the posterior (ischiofemoral) part of M. gluteus maximus, which is observed in the apes (Fig. 4).

Fig. 3. The lumbar and sacral plexus. (A) orangutan (B) chimpanzee. SGN: the superior gluteal nerve, IGN: the inferior gluteal nerve, SN: the sciatic nerve, Nif: the nerve branch of the sciatic nerve (the common peroneal nerve) to the ischiofemoral part, the long head of $\mathrm{M}$. biceps femoris, M. semitendinosus and $\mathrm{M}$. semimembranosus, $\mathrm{N}$ (red line): the nerve branch of the sciatic nerve (the common peroneal nerve) to the posterior part of $\mathrm{M}$. gluteus maximus, the long head of M. biceps femoris, M. semitendinosus and $\mathrm{M}$. semimembranosus. 

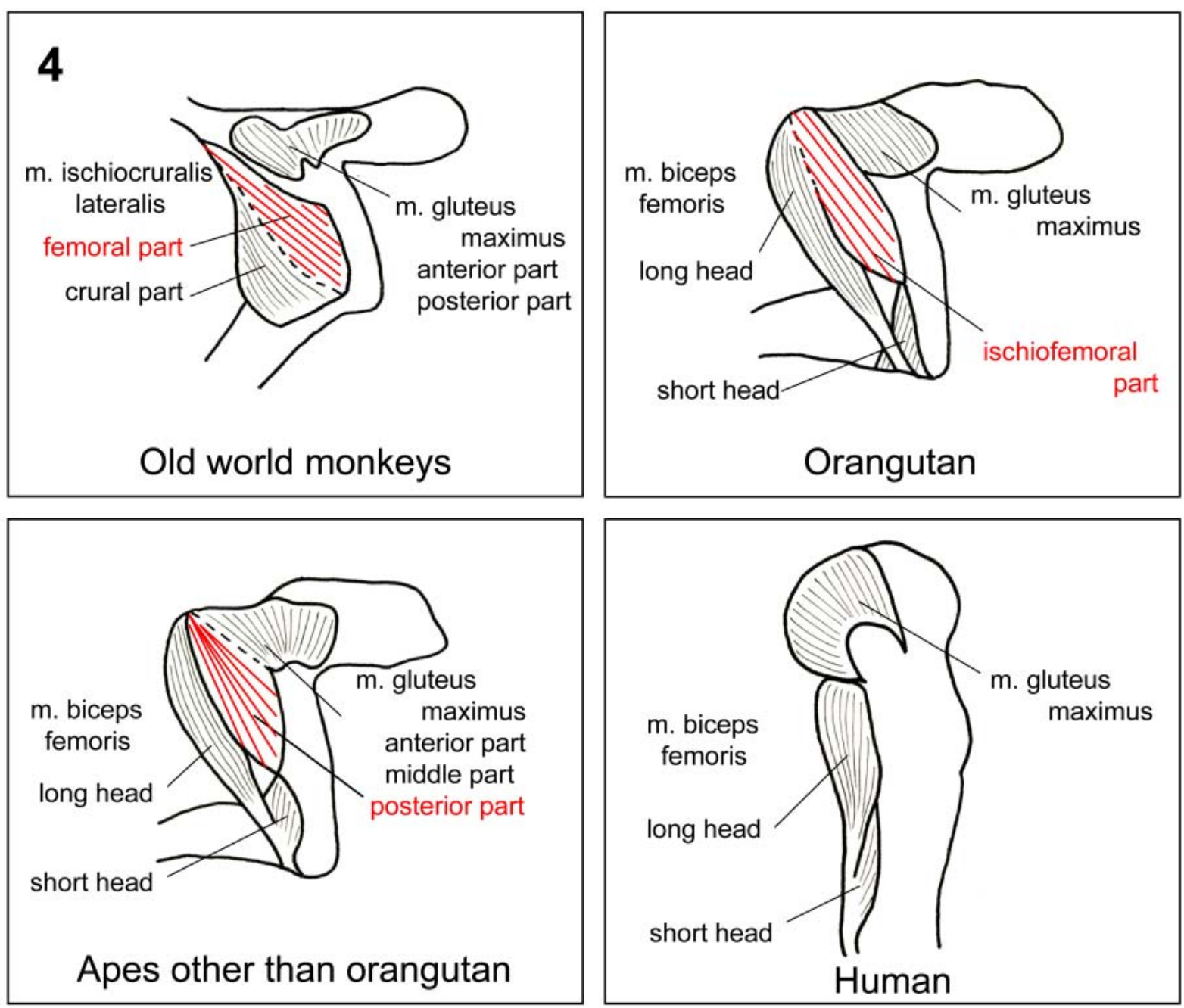

Fig. 4. Schematic drawing of M. biceps femoris and M. gluteus maximus in the old world monkeys, the orangutan, the apes other than the orangutan and human.

Therefore, it can be said that the form of M. biceps femoris and $\mathrm{M}$. gluteus maximus in the orangutan is unique, seeming to exist somewhere between that of the old world monkeys and the apes.

Conclusively, these obserevations and descriptions strongly suggest that the femoral part of M. biceps femoris (M. ischiocruralis lateralis) of the old world monkeys is equal to the ischiofemoral part of the long head of $\mathrm{M}$. biceps femoris of the orangutans, and be exchanged to the posterior (ischiofemoral) part of M. gluteus maximus in the other apes, however be quite different in structure in humans.

In addition, it is probable that these adaptations reflect the usage of lower extremities and the walking style of each primate. In the orangutan, the role of its lower extremities is similar to that of the upper extremities, where gripping or hanging is more important than pushing against the ground. Therefore, the development of the flexor complex of the thigh is necessary for the orangutan, resulting in a unique musculature of M. biceps femoris. It appears that this morphologic feature is an adaptation to arboreal life.

\section{REFERENCES}

1. Fick, R. 1895. Arch. f. Anat. U. Physiol. (Anat. Abth.): 1-100. (cited from Sonntag, C. F., 1924).

2. Hamada, Y. 1985. Primate hip and thigh muscles: Comparative anatomy and dry weights., pp. 131-152. In: Primate Morphophysiology, Locomotor Analyses and Human Bipedalism (Kondo, S. ed.), University of Tokyo Press, Tokyo.

3. Netter, F. H. 2003. pp. 476-485. Atlas of Human Anatomy, 3rd ed., Novartis Medical Education.

4. Raven, H. C. 1950. pp. 57-59. The Anatomy of the Gorilla, Columbia University Press, New York.

5. Sigmon, B. A. 1974. A functional analysis of pongid hip and thigh musculature. J. Hum. Evol. 3: 161-185 (cited from Hamada, Y., 1985).

6. Sigmon, B. A. 1976. Functions and evolution of hominoid hip and thigh musculature. pp. 235-252. In: Primate Functional 
Morphology and Evolution (Tuttle, R. H. ed.), Mouton, The Hague (cited from Hamada, Y., 1985).

7. Sonntag, C. F. 1924. pp. 349-450. On the Anatomy, Physiology and Pathology of the Orangutan. Proc Zool Soc, London.

8. Stern, J. T. Jr. 1971. Functional Myology of the Hip and Thigh of Cebid Monkeys and Its Implications for the Evolution of Erect Posture. Biblio Primatol No.14. S. Karger, Basel. (cited from Hamada, Y., 1985).
9. Swindler, D. R. and, Wood, C. D. 1973. pp. 254-261. An Atlas of Primate Gross Anatomy: Baboon, Chimpanzee, and Man, University of Washington Press, Washington.

10. Tuttle, R. H. and Cortright, G. W. 1988. Positional behavior, adaptive complexes, and evolution. pp. 311-330. In: Orangutan Biology (Jeffrey, H. S. ed.), Oxford University Press, New York. 